\title{
"Shape Activity": A Continuous-State HMM for Moving/Deforming Shapes With Application to Abnormal Activity Detection
}

\author{
Namrata Vaswani, Member, IEEE, Amit K. Roy-Chowdhury, and Rama Chellappa, Fellow, IEEE
}

\begin{abstract}
The aim is to model "activity" performed by a group of moving and interacting objects (which can be people, cars, or different rigid components of the human body) and use the models for abnormal activity detection. Previous approaches to modeling group activity include co-occurrence statistics (individual and joint histograms) and dynamic Bayesian networks, neither of which is applicable when the number of interacting objects is large. We treat the objects as point objects (referred to as "landmarks") and propose to model their changing configuration as moving and deforming "shape" (using Kendall's shape theory for discrete landmarks). A continuous-state hidden Markov model is defined for landmark shape dynamics in an activity. The configuration of landmarks at a given time forms the observation vector, and the corresponding shape and the scaled Euclidean motion parameters form the hidden-state vector. An abnormal activity is then defined as a change in the shape activity model, which could be slow or drastic and whose parameters are unknown. Results are shown on a real abnormal activity-detection problem involving multiple moving objects.
\end{abstract}

Index Terms-Abnormal acitivity detection, activity recognition, hidden Markov model (HMM), landmark shape dynamics, particle filtering, shape activity.

\section{INTRODUCTION}

$\mathbf{I}$ $\mathrm{N}$ THIS paper, we develop models for the configuration dynamics of a group of moving landmarks (point objects) in shape space. Shape of a group of discrete points (known as "landmarks") is defined by Kendall [1] as all the geometric information that remains when location, scale and rotational effects (referred to as "motion parameters" in this paper) are filtered out. There has been a lot of work in learning the statistics of a dataset of similar shapes and defining probability distributions in shape and preshape space, [2] provides a good overview. Statistical shape theory began in the late 1970s and has evolved into viable statistical approaches for modeling the shape of an object with applications in object recognition and matching. In this work, we extend these static classification

Manuscript received August 22, 2003; revised April 5, 2004. This work was supported in part by the DARPA/ONR under Grant N00014-02-1-0809. The associate editor coordinating the review of this manuscript and approving it for publication was Dr. Michel Schmitt.

$\mathrm{N}$. Vaswani is with the Department of Electrical and Computer Engineering, Iowa State University, Ames, IA 50011 USA (e-mail: namrata@iastate.edu).

A. K. Roy-Chowdhury is with the Department Electrical Engineering, University of California, Riverside, CA 92521 USA (e-mail: amitrc@ee.ucr.edu).

R. Chellappa is with the Department of Electrical and Computer Engineering and the Center for Automation Research, University of Maryland, College Park, MD 20742 USA (e-mail: rama@ cfar.umd.edu).

Digital Object Identifier 10.1109/TIP.2005.852197 approaches to defining dynamical models for landmark shape deformation. Also, we consider here the shape formed by a configuration of point objects instead of that of a single object.

For a dataset of similar shapes, the shape variability can be modeled in the tangent hyperplane to the shape space at the mean shape [2]. The tangent hyperplane is a linearized version of the shape space linearized at a particular point known as the pole of tangent projection. Typically, one uses the Procrustes mean [2] of the dataset as the pole. The tangent plane is a vector space, and, hence, techniques from linear multivariate statistics can be used to model shape variability in tangent space. In this work, we model shape dynamics by defining an autoregressive (AR) model in the tangent plane at the mean shape. To model the configuration dynamics, we also define motion models (models for translation, isotropic scaling and rotation). We use the term "shape activity" to denote a continuous-state hidden Markov model (HMM) (also referred to as a "partially observed nonlinear dynamical model" or a "stochastic-state space model" in different contexts) for the shape deformation and motion in the activity.

Previous approaches to modeling activity performed by groups of point objects include co-occurrence statistics (e.g., [3]) and discrete-state dynamic Bayesian networks (DBNs) (e.g., [4]). Co-occurrence statistics involves learning individual and joint histograms of the objects. Joint histograms for modeling interactions is feasible only when the number of interacting objects is small. Our approach on the other hand implicitly models interactions and independent motion of a group of objects with any number of interacting objects. DBNs define high level relations between different events and typically use heuristics for event detection. Our algorithms can be used to provide a more principled strategy for event detection using DBNs. Another advantage of our framework is that using shape and its dynamics makes the representation invariant to translation, in-plane rotation or sensor zoom. The idea of using "shape" to model activities performed by groups of moving objects is similar to recent work in literature on controlling formations of groups of robots using shape (e.g., [5]).

One example of a stationary shape activity, that we discuss in this paper, is that of people (treated as point-objects) deplaning and moving toward the terminal at an airport [see Fig. 2(a)]. Our framework can be used to model normal activity and detect abnormal activity as a deviation from the normalcy model. We are able to detect both spatial and temporal abnormalities (terminology borrowed from [3]). The "landmark" could also be a moving vehicle and one could model traffic in a certain 
region as the normal activity and define lane change as the "abnormality." Our framework can also be used to model the dynamics of articulated shapes like the human body (the different rigid parts of the human body forming the landmarks) and, thus, represent different actions [6]. This has application in classifying or tracking a sequence of actions and also in detecting motion disorders. Also, our approach is sensor independent. The same framework could be used for point location observations obtained from other sensors, e.g., infrared, acoustic, radar, or seismic, and only the observation model would change.

\section{A. Organization of the Paper}

This paper is organized as follows. We discuss related work in the next subsection. Some definitions and methods for shape analysis are presented in Section II. The shape dynamics for stationary shape activity and the training algorithm to learn its parameters is described in Section III-A. The noise in the observed configuration makes the state (shape, motion) partially observed (or hidden). The partially observed model is discussed in Section III-B. The nonstationary shape activity model is given in Section III-C. The particle filtering algorithm to estimate the hidden state from the observations is discussed in Section III-D and its advantages are discussed in Section III-E. The abnormality-detection problem and its formulation as a change-detection problem is discussed in Section IV. The strategy to deal with time-varying number of landmarks is given in Section V. Experimental results on the airport terminal abnormal activity-detection problem are presented in Section VI. Extensions of our framework to tracking observations and to activity sequence identification and tracking are discussed in Section VII. Conclusions are given in Section VIII.

\section{B. Related Work}

Shape Representations: Some of the commonly used representations for shape are Fourier descriptors [7], splines [8], and deformable snakes all of which model the shape of continuous curves, but, in our work, we are attempting to model the dynamics of a group of discrete landmarks (which could be moving point-objects or moving parts of an articulated object like the human body). Since the data is inherently finite dimensional, using infinite-dimensional representations of a continuous curve is not necessary, and, hence, we look only at the representation of shape in $\Re^{n}$ (modulo Euclidean similarity transformations), which was first defined by Kendall in 1977. Active shape models introduced by Cootes et al. [9] also consider the shape of points in $\Re^{n}$. In [10], they define point distribution models which are principal component models for shape variation using Procrustes residuals.

Modeling Shape Change: There has been a lot of work in defining probability distributions in (Kendall's) shape and preshape space and also in analyzing datasets of similar shapes in the tangent space at the mean (discussed in [2, Ch. 6, 7, and 11], [11]-[13], and references therein). Many models for shape deformation of one shape into another have been proposed which include affine deformation, thin plate splines, and principal and partial warp deformations (discussed in [2, Ch. 10]), but none of these define dynamical models for time seqeunces of shapes. We propose in this paper, a partially observed dynamical model (which also satisfies the HMM property, and, hence, we refer to it as an HMM in the rest of the paper) for stationary and nonstationary shape activities. Our model for nonstationary shape activities is similar in spirit to those in [14] and [15] where the authors define dynamical models for motion on Lie groups and Grassmann manifolds, respectively, using piecewise geodesic priors and track them using particle filtering.

Modeling Activity: There is a huge body of work in computer vision on modeling and recognition of activities, human actions and events. The work can be classified (based on the formalisms used) as Bayesian networks (BNs) and DBNs [16], [4]; finite-state HMMs for representing activity [17], [18]; stochastic grammars [19]; and factorization method based approaches [20], [21]. In [3], the authors perform clustering to learn the co-occurrence statistics of individual objects and their interactions with other objects. [22] is another work which treats events as long spatiotemporal objects and clusters them based on their behavioral content. In [23], action "objects" are represented using generalized cylinders with time forming the cylinder axis. Now, [3] and [20]-[23] are nonparametric approaches to activity/event recognition, while HMMs, stochastic grammars, BNs, and DBNs are model based approaches. Our work also defines a parametric model (but it is a continuous-state HMM) for activity performed by a group of objects and there are some other differences. First, we treat objects as point-objects, and, hence, we can get our observations from low resolution video or even from other sensors like radar, acoustic, or infrared. Second, we provide a single global framework for modeling the interactions and independent motion of multiple moving objects by treating them as a deformable shape.

Particle Filters (PFs) and Change Detection: PFs [24] have been used extensively in computer vision for tracking a single moving object in conjunction with a measurement algorithm to obtain observations [25]-[27]. In [28], particle filtering is used to track multiple moving objects but they use separate state vectors for each object and define data association events to associate the state and observation vectors. In this paper, we represent the combined state of all moving objects using the shape and global motion of their configuration and define a dynamic model for both shape and motion. We use a PF to filter out the shape from noisy observations of the object locations and use the filtered shape for abnormal activity detection. We define an abnormal activity as a change which could be slow or drastic and whose parameters are unknown. An algorithm for change detection in nonlinear systems using PFs is given in [29], but it assumes that the changed system's parameters are known and it deals only with sudden changes. In this paper we use a statistic called ELL for detecting slow changes, with unknown parameters [30], [31].

\section{PRELIMINARIES AND NotATION}

We would first like to clarify that the terms partially observed dynamical model and HMM are used interchangeably for "shape activity" models since the partially observed dynamic model that we define is also an HMM. We use "arg" to denote the angle of a complex scalar as well as in "arg min" for the argument minimizing a function, but the meaning is clear from 
the context. ${ }^{*}$ is used to denote conjugate transpose. $\|$.$\| is$ used for the Euclidean norm of a complex or real vector and |.| for the absolute value of a complex scalar. $I_{k}$ denotes the $k \times k$ identity matrix and $1_{k}$ denotes a $k$-dimensional vector of ones. Also note that, to simplify notation, we do not distinguish between a random process and its realization. We review below the tools for statistical shape analysis as described in [2].

Definition 1: [2] The configuration is an ordered set ( $k$-tuple) of landmarks (which in our case is the $k$-tuple of point-object locations). The configuration matrix is a $k \times m$ matrix of Cartesian coordinates of the $k$ landmarks in $m$ dimensions. For two-dimensional data $(m=2)$, a more compact representation is a $k$-dimensional complex vector with $x$ and $y$ coordinates forming the real and imaginary parts. The configuration space is the space of all $k$-tuples of landmarks, i.e., $\Re^{k m}$.

Translation Normalization: The complex vector of the configuration $\left(Y_{\text {raw }}\right)$ can be centered by subtracting out the centroid of the vector, thus yielding a centered configuration, i.e.,

$$
Y=C Y_{\text {raw }} \quad \text { where } \quad C=I_{k}-\frac{1_{k} 1_{k}^{T}}{k} .
$$

Definition 2: [2] The preshape of a configuration matrix (or complex vector) $Y_{\text {raw }}$ is all the geometric information about $Y_{\text {raw }}$ that is invariant under location and isotropic scaling. The preshape space $S_{m}^{k}$ is the space of all possible preshapes. $S_{m}^{k}$ is a hyper-sphere of unit radius in $\Re^{(k-1) m}$, and, hence, its dimension is $(k-1) m-1$ (a unit hyper-sphere in $\Re^{P}$ has dimension $P-1)$.

Scale Normalization: The preshape is obtained by normalizing the centered configuration, $Y$, by its Euclidean norm, $s(Y)=\|Y\|$ (known as scale or size of the configuration), i.e., $w(Y)=Y / s(Y)$.

Definition 3: [2] The shape of a configuration matrix (or complex vector) $Y_{\text {raw }}$ is all the geometric information about $Y_{\text {raw }}$ that is invariant under location, isotropic scaling, and rotation, i.e., $[z]=\left\{s Y_{\text {raw }} R+1_{k} \alpha^{T}: s \in \Re^{+}, R \in S O(m), \alpha \in\right.$ $\left.\Re^{m}\right\}$. The shape space is the set of all possible shapes. Formally, the shape space $\Sigma_{m}^{k}$ is the orbit space of the noncoincident $k$ point set configurations in $\Re^{m}$ under the action of Euclidean similarity transformations. The dimension of shape space is $M=(k-1) m-1-m(m-1) / 2$. It is easy to see that $\Sigma_{m}^{k}=S_{m}^{k} / S O(m)$, i.e., $\Sigma_{m}^{k}$ is the quotient space of $S_{m}^{k}$ under the action of the special orthogonal group of rotations $S O(\mathrm{~m})$.

Rotation Normalization: Shape $z$ is obtained from a preshape $w$ by rotating it in order to align it to a reference preshape $\gamma$. The optimal rotation angle is given by $\theta(Y, \gamma)=\arg \left(w^{*} \gamma\right)=\arg \left(Y^{*} \gamma\right)$, and the shape, $z(Y, \gamma)=w e^{j \theta(Y, \gamma)}=(Y / s(Y)) e^{j \theta(Y, \gamma)}$.

In this paper, we deal with $m=2$-dimensional shapes, and, hence, the configuration vector is represented as a $k$-dimensional complex vector and the shape space dimension is $(2 k-4)$.

Distance Between Shapes: A concept of distance between shapes is required to fully define the non-Euclidean shape metric space. We use the Procrustes distance which is defined as follows.

\section{Definition 4: [2] The full Procrustes fit of $w$ onto $y$ is}

$$
\begin{aligned}
w^{P}(y) & =\hat{\beta} e^{j \hat{\theta}} w+\hat{a}+j \hat{b} \quad \text { where } \\
\hat{\beta}, \hat{\theta}, \hat{a}, \hat{b} & =\arg \min _{(\beta, \theta, a, b)} D(y, w) \\
D(y, w) & =\left\|y-\left(\beta e^{i \theta} w+a+j b\right)\right\| .
\end{aligned}
$$

If $y$ and $w$ are preshapes, it is easy to see that the matching parameters are [2, result 3.1] $\hat{a}+j \hat{b}=0, \hat{\theta}=\arg \left(w^{*} y\right), \hat{\beta}=$ $\left|w^{*} y\right|=\left(y^{*} w w^{*} y\right)^{1 / 2}$.

Definition 5: [2] The full Procrustes distance between preshapes $w$ and $y$ is the Euclidean distance between the Procrustes fit of $w$ onto $y$, i.e.,

$$
\begin{aligned}
D_{F}(w, y) & =\inf _{\beta, \theta, a, b} D(y, w)=\left\|y-w^{P}(y)\right\| \\
& =\sqrt{1-y^{*} w w^{*} y} .
\end{aligned}
$$

Definition 6: [2] The full Procrustes estimate of mean shape (commonly referred to as full Procrustes mean) of a set of preshapes $\left\{w_{i}\right\}$ is the minimizer of the sum of squares of full Procrustes distances from each $w_{i}$ to an unknown unit size mean configuration $\mu$, i.e.,

$$
\begin{aligned}
{[\hat{\mu}] } & =\arg \min _{\mu:\|\mu\|=1} \sum_{i=1}^{n} \min _{\beta_{i}, \theta_{i}, a_{i}, b_{i}} D^{2}\left(w_{i}, \mu\right) \\
& =\arg \min _{\mu:\|\mu\|=1} \sum_{i=1}^{n} D_{F}^{2}\left(w_{i}, \mu\right) \\
& =\arg \min _{\mu:\|\mu\|=1} \sum_{i=1}^{n}\left(1-\mu^{*} w_{i} w_{i}^{*} \mu\right) \\
& =\arg \max _{\mu:\|\mu\|=1} \mu^{*}\left[\sum_{i=1}^{n} w_{i} w_{i}^{*}\right] \mu
\end{aligned}
$$

i.e., $[\hat{\mu}]$ is given by the set of complex eigenvectors corresponding to the largest eigenvalue of $S \triangleq \sum_{i=1}^{n} w_{i} w_{i}^{*}[2$, result 3.2].

Shape Variability in Tangent to Shape Space: The structure of shape variability of a dataset of similar shapes can be studied in the tangent space to the shape space. We shall consider the tangent projections to the preshape sphere after normalizing for rotation (w.r.t. the pole), which form a suitable tangent coordinate system for shape. The tangent space is a linearized local approximation of shape space at a particular point in shape space which is called the pole of tangent projection. Thus, Euclidean distance in tangent space is a good approximation to Procrustes distance, for points in the vicinity of the pole (see [2, Ch. 4] for more details).

Definition 7: [2] The Procrustes tangent coordinates of a centered configuration, $Y$, taking $\mu$ as the pole, are obtained by projecting $z(Y, \mu)$ (the shape of $Y$ aligned to $\mu$ ) into the tangent space at $\mu$, i.e.,

$$
v(Y, \mu)=\left[I_{k}-\mu \mu^{*}\right] z(Y, \mu)=\left[I_{k}-\mu \mu^{*}\right] \frac{Y}{s(Y)} e^{j \theta(Y, \mu)} .
$$

The inverse of the above mapping (tangent space to centered configuration space) is

$$
Y(v, \theta, s, \mu)=\left[\left(1-v^{*} v\right)^{1 / 2} \mu+v\right] s e^{-j \theta} .
$$




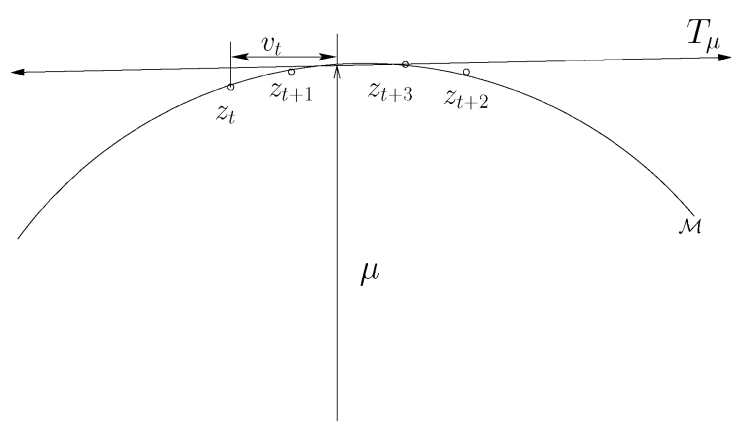

(a) Stationary Shape Activity (SSA)

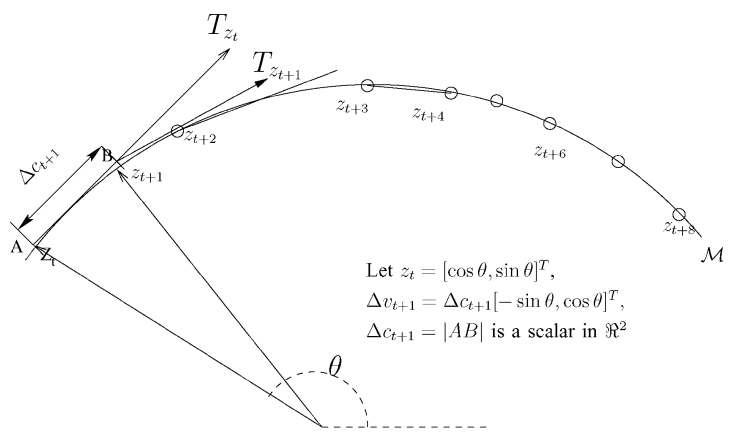

(b) Nonstationary Shape Activity (NSSA)

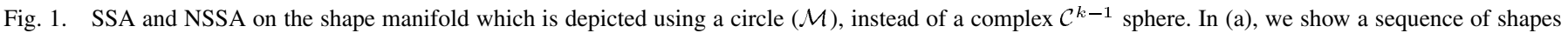

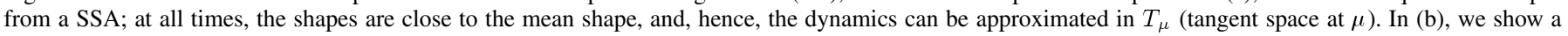
sequence of shapes from an NSSA, the shapes move on the shape manifold, and, hence, we need to define a new tangent space at every time instant.

The shape space is a manifold in $\mathcal{C}^{k-1}$, and, hence, its dimension is $k-2$. Thus, the tangent space at any point of the shape space is a $k$ - 2-dimensional hyperplane in $\mathcal{C}^{k}$ (or, equivalently, a $(2 k-$ 4)-dimensional hyperplane in $\Re^{2 k}$ ) [2].

\section{MOdeling SHAPE DyNAMICS}

The distinction between motion and deformation of a deformable shape is not clear. We separate the dynamics of a deforming configuration into scaled Euclidean motion (translation, rotation, and uniform scaling) of the mean shape and nonrigid deformations. This idea is similar to that suggested in [32] for continuous curves. We define a continuous-state HMM for the changing configuration of a group of moving landmarks (point-objects) with the shape and scaled Euclidean motion parameters being the hidden-state variables and the noisy configuration vector forming the observation. We refer to it as a "shape activity." A "stationary shape activity" is defined as one for which the shape vector is stationary, i.e., the mean shape ${ }^{1}$ remains constant with time and the deformation model is stationary while in a "nonstationary shape activity," the mean shape changes with time.

We discuss below the stationary and nonstationary shape activity models and also the particle filtering algorithm to estimate the shape from the noisy configuration observations. The entire discussion assumes a fixed number of landmarks, but in certain applications like the airport scenario with people deplaning, the number of landmarks varies with time. We deal with this by resampling the curve formed by joining the landmarks to a fixed number of points. This is discussed in Section V. Also, note that in this representation of the shape of discrete landmarks, correspondences between landmarks are assumed to be known across frames. Since the number of landmarks is usually small ( $k=8$ in this case), this is easy to ensure.

\section{A. Stationary Shape Activity: Shape Deformation Model in Tangent Space}

A sequence of point configurations from a stationary shape activity (SSA), with small system noise variance, would lie close to each other and to their mean shape [see Fig. 1(a)].

\footnotetext{
${ }^{1}$ In the entire paper, "mean shape" is used to refer to the full Procrustes mean calculated over samples from the given probability distribution.
}

Hence, a single tangent space at the mean is a good approximate linear space to learn the shape deformation dynamics for a SSA. We represent a configuration of landmarks by a complex vector with the $x$ and $y$ coordinates of a landmark forming the real and imaginary parts. $^{2}$ We discuss the training algorithm, i.e., how to learn the shape dynamics given a single training sequence of configurations. Given a sequence of configurations with negligible observation noise $\left\{Y_{\text {raw }, t}\right\}$, we learn its Procrustes mean and evaluate the tangent coordinates of shape (using the Procrustes mean as the pole), as follows:

$$
\begin{aligned}
Y_{t} & =C Y_{\text {raw }, t} \\
s_{t} \triangleq s\left(Y_{t}\right) & =\left\|Y_{t}\right\|, \quad w_{t}=\frac{Y_{t}}{s_{t}} \\
\mu & =\arg \max _{\mu:\|\mu\|=1} \mu^{*}\left[\sum_{t=1}^{T} w_{t} w_{t}^{*}\right] \mu \\
\theta_{t} \triangleq \theta\left(Y_{t}, \mu\right) & =\arg \left(w_{t}^{*} \mu\right), \quad z_{t}=w_{t} e^{j \theta_{t}} \\
v_{t} \triangleq v\left(Y_{t}, \mu\right) & =\left[I_{k}-\mu \mu^{*}\right] z_{t}=\left[I_{k}-\mu \mu^{*}\right] \frac{Y_{t} e^{j \theta_{t}}}{s_{t}} .
\end{aligned}
$$

Since the tangent coordinates are evaluated w.r.t. the mean shape of the data, assuming that they have zero mean is a valid assumption. We string the complex tangent vector components as a $2 k$-dimensional real vector and define a linear Gauss-Markov model on it to model the shape deformation dynamics. Note that since we are assuming small variations about a mean shape, a first-order Gauss-Markov model is sufficient to model the shape dynamics in this case, i.e.,

$$
\begin{aligned}
v_{t} & =A_{t} v_{t-1}+n_{t} \\
v_{0} & \sim \mathcal{N}\left(0, \Sigma_{v, 0}\right), \quad n_{t} \sim \mathcal{N}\left(0, \Sigma_{n, t}\right)
\end{aligned}
$$

where $\left\{n_{t}\right\}$ is i.i.d. Gaussian system noise. The deformation process is assumed to be stationary and ergodic. Under this assumption the above is a first-order autoregressive (AR) model. Thus, $\Sigma_{v, 0}=\Sigma_{v, t}=\Sigma_{v}, \Sigma_{n, t}=\Sigma_{n}$ and $A_{t}=A$ is the autoregression matrix with $A<I$. Thus, all the three parameters

${ }^{2}$ Note that all transformations between the configuration space to shape space and tangent to shape space are defined in $\mathcal{C}^{k}$ ( $k$-dimensional complex space) but the dynamical model on tangent coordinates is defined in $\Re^{2 k}$ by vectorizing the complex vector. This is done only for compactness of representation. The entire analysis could instead have been done in $\Re^{2 k}$. 
can be learned using a single training sequence of tangent coordinates, $\left\{v_{t}\right\}$, as follows [33]:

$$
\begin{aligned}
A & =R_{v}(1) \Sigma_{v}^{-1}, \quad \text { where } \\
\Sigma_{v} & =\frac{1}{T} \sum_{t=1}^{T} v_{t} v_{t}^{T}, \quad \text { and } \quad R_{v}(1)=\frac{1}{T-1} \sum_{t=2}^{T} v_{t} v_{t-1}^{T} \\
\Sigma_{n} & =\frac{1}{T} \sum_{t=1}^{T}\left(v_{t}-A v_{t-1}\right)\left(v_{t}-A v_{t-1}\right)^{T}
\end{aligned}
$$

and the joint pdf of $v_{t}$ is given by

$$
\begin{aligned}
p\left(v_{t}\right) & =\mathcal{N}\left(0, \Sigma_{v}\right), \quad \forall t \\
p\left(v_{t} \mid v_{t-1}\right) & =\mathcal{N}\left(A v_{t-1}, \Sigma_{n}\right), \quad \forall t .
\end{aligned}
$$

Note that the asymptotically stationary case where $A<I$ but $\Sigma_{v, 0} \neq \Sigma_{v}$, so that $\Sigma_{v, t} \rightarrow \Sigma_{v}$, only for large time instants $(t \rightarrow \infty)$, can also be dealt with in the above framework. In that case, $\Sigma_{v, 0}$ is defined using a priori knowledge, $\Sigma_{n}$ can be learned exactly as in (9), and $\Sigma_{v}, R_{v}(1)$ can also be learned as in (9), but by excluding the summation over the initial (transient) time instants.

\section{B. Stationary Shape Activity: Partially Observed (Hidden) Shape Dynamics}

In the previous subsection, we defined a dynamic model on the shape of a configuration of moving points. We assumed that the observation sequence used for learning the shape dynamics has zero (negligible) observation noise associated with it (e.g., if it were hand picked), but a test sequence of point configurations $\left\{Y_{\text {raw }, t}\right\}$ will usually be obtained automatically using a measurement algorithm (e.g., a motion-detection algorithm [34]). It will, thus, have large observation noise associated with it, i.e., $Y_{\text {raw }, t}=Y_{\text {raw }, t}^{\text {actual }}+\zeta_{\text {raw }, t}$, where $\zeta_{\text {raw }, t}$ is zero mean i.i.d. Gaussian noise, $\zeta_{\text {raw }, t} \sim \mathcal{N}\left(0, \Sigma_{\text {obs,raw }, t}\right)$. If the different landmarks are far apart, the noise can be assumed to be i.i.d. over the different landmarks as well (i.e., white $\Sigma_{\text {obs,raw }, t}$ ). Now, translation normalization is a linear process, and, hence, $Y_{t}=C Y_{\text {raw }, t}$ is also Gaussian ${ }^{3}$ with observation noise $\zeta_{t}$, given by

$$
\Sigma_{\mathrm{obs}, t}=C \Sigma_{\mathrm{obs}, \mathrm{raw}, t} C^{T}
$$

[ $C$ is the centering matrix defined in (1)], but the mapping from centered configuration space to the tangent space is nonlinear (scaling by $\left\|Y_{t}\right\|$ followed by rotation to align with mean), and, hence, it is not possible to obtain a closed-form expression for the pdf of the tangent coordinates given that there is observation noise in the configuration vector. To deal with this, one solution is to define a partially observed dynamical model which can then be tracked using a PF to estimate the distribution of the tangent coordinates of shape given the noisy observations. The observed centered configuration, $Y_{t}$, forms the observation vector and the shape, scale and rotation form the hidden-state vector. We discuss the PF in Section III-D and its advantage over an EKF in Section III-E.

\footnotetext{
${ }^{3}$ Note that, here, we have assumed Gaussian observation noise $\zeta_{\text {raw }, t}$, but, in general, a PF can track with any kind of noise; however, for non-Gaussian $\zeta_{\text {raw }, t}$, it is, in general, not possible to define a distribution for $\zeta_{t}$, and one would have to treat the translation as part of the state vector.
}

Now, we have the following observation model for a "stationary shape activity" with the observation vector $Y_{t}$ being the centered configuration vector and the state vector $X_{t}=$ $\left[v_{t}, s_{t}, \theta_{t}\right]$

$$
\begin{aligned}
Y_{t} & =h\left(X_{t}\right)+\zeta_{t}, \quad \zeta_{t} \sim \mathcal{N}\left(0, \Sigma_{\mathrm{obs}, t}\right) \\
h\left(X_{t}\right) & \left.=z_{t} s_{t} e^{-j \theta_{t}}, \text { where } z_{t}=\left(1-v_{t} * v_{t}\right)^{1 / 2}\right] \mu+v_{t} .
\end{aligned}
$$

Defining scale and rotation (motion parameters) as part of the state vector implies that we need to define prior dynamic models for them (motion model). The motion model can be defined based on either the motion of the shape if it is a moving configuration or based on motion of the measurement sensor if the sensor is moving (e.g., a moving camera or just an unstable camera undergoing a slight random motion) or a combined effect of both. A camera on an unstable platform, like an unmanned air vehicle (UAV), will have small random $x-y$ motion (translation), motion in $z$ direction (scale change) and rotation about the $z$ axis (rotation angle change). The translation gets removed when centering $Y_{\text {raw }, t}$. The scale and rotation can be modeled in this case by using an AR model both for log of scale and for the unwrapped rotation angle ${ }^{4}$, i.e.,

$$
\begin{aligned}
\log s_{t} & =\alpha_{s} \log s_{t-1}+\left(1-\alpha_{s}\right) \mu_{s}+n_{s, t} \\
\log s_{0} & \sim \mathcal{N}\left(\mu_{s}, \sigma_{s}^{2}\right), \quad n_{s, t} \sim \mathcal{N}\left(0, \sigma_{r}^{2}\right) \\
\theta_{t} & =\alpha_{\theta} \theta_{t-1}+\left(1-\alpha_{\theta}\right) \mu_{\theta}+n_{\theta, t} \\
\theta_{0} & \sim \mathcal{N}\left(0, \sigma_{\theta}^{2}\right), \quad n_{\theta, t} \sim \mathcal{N}\left(\mu_{\theta}, \sigma_{\theta}^{2}\right) .
\end{aligned}
$$

The motion model parameters can be learned using the training sequence values of $\left\{s_{t}\right\}_{t=1}^{T}$ and $\left\{\theta_{t}\right\}_{t=1}^{T}$ given by (6). $\left\{\theta_{t}\right\}_{t=1}^{T}$ will have to be the unwrapped value of the rotation angle to learn a Gaussian model. Also, one can either assume wide sense stationarity, in which case $\mu_{s}, \sigma_{s}^{2}, \sigma_{r}^{2}, \alpha_{s}$ and $\mu_{\theta}, \sigma_{\theta}^{2}, \sigma_{u}^{2}, \alpha_{\theta}$ can be learned using Yule-Walker equations [33], or assume a random walk motion model(set $\alpha_{s}=1$ and $\alpha_{\theta}=1$ ), depending on the application.

The shape deformation dynamics [(8) in Section III-A] and the motion model defined above (13) form the system model while (12) defines the observation model. Thus, we have defined a continuous-state HMM (partially observed dynamic model) for a "stationary shape activity." The model is nonlinear since the mapping $h\left(X_{t}\right)$ is nonlinear.

\section{Nonstationary Shape Dynamics}

For a "nonstationary shape activity" model (first proposed in [6] and [39]), the mean shape is time varying and, hence, modeling the shape dynamics requires a time-varying tangent space [see Fig. 1(b)] defined with the current shape as the pole. Note that, modulo reflections, there is a one-to-one mapping between the tangent space at any point on the shape manifold and the shape manifold, but the distance between two points on a tangent plane is a good approximation to the distance on the shape manifold only for points close to the pole of the tangent plane. Hence, the assumption of i.i.d. system noise to go from shape at $t$ to shape at $t+1$ is valid only for shapes in the vicinity

\footnotetext{
${ }^{4}$ Since we are modeling only random motion of a camera, a first-order linear Markov model for log of scale and rotation is sufficient in this case.
} 
of the pole. Thus, when the shape variation is large (for NSSA), there is a need to define a tangent space with the current shape being the pole.

The state space now consists of the mean shape at time $t$, $z_{t}$, the "shape velocity coefficients" vector $c_{t}$ and the motion parameters (scale $s_{t}$, rotation $\theta_{t}$ ), i.e., state $X_{t}=\left[z_{t}, c_{t}, s_{t}, \theta_{t}\right]$. Denote the tangent space at $z_{t}$ by $T_{z_{t}}$. We then have the following dynamics: The tangent coordinate of $z_{t}$ in $T_{z_{t-1}}$ [denoted by $\left.v_{t}\left(z_{t}, z_{t-1}\right)\right]$ defines a "shape velocity" (time derivative of shape) vector. We perform a singular value decomposition [33] of the tangent projection matrix $\left[I_{k}-z_{t-1} z_{t-1}^{*}\right] C$ to obtain an orthogonal basis for the $(k-2)$-dimensional tangent hyperplane $T_{z_{t-1}}$. Denote the orthogonal basis matrix for $T_{z_{t-1}}$ by $U\left(z_{t-1}\right) .{ }^{5}$ The $(k-2)$-dimensional vector of coefficients along these basis directions, denoted by $c_{t}\left(z_{t}, z_{t-1}\right)$, is a coefficients vector for the "shape velocity" $v_{t}$, i.e., $v_{t}=U\left(z_{t-1}\right) c_{t}$. The shape at $t, z_{t}$ is obtained by "moving" $z_{t-1}$ on the shape manifold as follows: "Move" an amount $v_{t}$ (from origin) in $T_{z_{t-1}}$ and then project back onto shape space. This is done as follows: $z_{t}=\left(1-v_{t}^{*} v_{t}\right)^{1 / 2} z_{t-1}+v_{t}$.

We define a linear Gauss-Markov model on shape velocity $v_{t}$ which corresponds to a linear Gauss-Markov model for $c_{t}$. We can then summarize the shape dynamics as follows:

$$
\begin{aligned}
& c_{t}=A_{c, 2, t} c_{t-1}+n_{t}, \quad n_{t} \sim \mathcal{N}\left(0, \Sigma_{n, c, 2, t}\right) \\
& v_{t}=U\left(z_{t-1}\right) c_{t}, \quad U\left(z_{t-1}\right)=\text { orthogonal basis }\left(T_{z_{t-1}}\right) \\
& z_{t}=\left(1-v_{t}^{*} v_{t}\right)^{1 / 2} z_{t-1}+v_{t} .
\end{aligned}
$$

If we assume a time invariant $\mathrm{AR}$ model on $\left\{v_{t}\right\}$, i.e., $v_{t}=$ $A_{v, 2} v_{t-1}+n_{v, t}$, then we have a time-varying Gauss-Markov model on $c_{t}$ with

$$
\begin{aligned}
A_{c, 2, t} & =U\left(z_{t-1}\right)^{*} A_{v, 2} U\left(z_{t-2}\right), \quad \text { and } \\
\Sigma_{n, c, 2, t} & =U\left(z_{t-1}\right)^{*} \Sigma_{n, v, 2} U\left(z_{t-2}\right) .
\end{aligned}
$$

Note that a Markov model on the shape velocity corresponds to a second-order Markov model on shape, $z_{t}$ (hence, the subscript " 2 " on the parameters). Some special cases are $A_{v, 2}=0$ or i.i.d. velocity (first-order Markov model on shape), $A_{v, 2}=I$ which corresponds to i.i.d. shape acceleration and $A_{v, 2}=A_{A R}$ or stationary shape velocity.

The motion model (model on $s_{t}, \theta_{t}$ ) can be defined exactly as in (13), but, now, $\theta_{t}$ is the rotation angle of current configuration w.r.t. the current mean shape $\mu_{t}=z_{t-1}$, and, hence, is a measure of rotation speed. As before, one can assume the motion model to be stationary or nonstationary. The shape and motion model, (14) and (13)), form the system model. The observation model is as follows:

$$
Y_{t}=\tilde{h}\left(X_{t}\right)+\zeta_{t}, \quad \text { where } \tilde{h}\left(X_{t}\right)=z_{t} s_{t} e^{-j \theta_{t}}
$$

\footnotetext{
${ }^{5}$ The basis vectors $\left\{\underline{u}_{t, i}\right\}_{i=1}^{k-2}$ are arranged as column vectors of a matrix $U\left(z_{t-1}\right)$, i.e., $U_{t}^{k \times(k-2)}=\left[\underline{u}_{t, 1}, \underline{u}_{t, 2} \cdots \underline{u}_{t, k-2}\right]$. $U_{t}^{k \times(k-2)}=$ orthogonal basis $\left(T_{z_{t-1}}\right)$ is evaluated as $U_{t}=U_{\text {full }, t} Q$, where $U_{\text {full }, t} S U_{\text {full }, t}^{*}=\left[I_{k}-z_{t-1} z_{t-1}^{*}\right] C$ and $Q=\left[I_{(k-2) \times(k-2)}, 0_{(k-2) \times 2}\right]^{T}$
}

1) Training: Given a training sequence of centered (translation normalized) configurations, $\left\{Y_{t}\right\}_{t=1}^{T}$, we first evaluate $\left\{c_{t}, v_{t}, s_{t}, \theta_{t}\right\}_{t=1}^{T}$ as follows: ${ }^{6}$

$$
\begin{aligned}
s_{t} & =\left\|Y_{t}\right\|, \quad w_{t}=\frac{Y_{t}}{s_{t}} \\
\theta_{t}\left(Y_{t}, z_{t-1}\right) & =\arg \left(w_{t}^{*} z_{t-1}\right), \quad z_{t}\left(Y_{t}, z_{t-1}\right)=w_{t} e^{j \theta_{t}} \\
v_{t}\left(Y_{t}, z_{t-1}\right) & =\left[I_{k}-z_{t-1} z_{t-1}^{*}\right] z_{t} \\
c_{t}\left(Y_{t}, z_{t-1}\right) & =U\left(z_{t-1}\right)^{*} z_{t} .
\end{aligned}
$$

Assuming a time invariant AR model on shape velocity $v_{t}$, one can learn its parameters $\left(A_{v, 2}, \Sigma_{n, v, 2}\right)$, as in (9), and then define the time-varying Markov model for $c_{t}$ using (15).

\section{Particle Filtering Algorithm}

The problem of nonlinear filtering is to compute at each time $t$, the conditional probability distribution, of the state $X_{t}$ given the observation sequence $Y_{1: t}=\left(Y_{1}, Y_{2}, \ldots, Y_{t}\right)$, $\pi_{t}(d x)=\operatorname{Pr}\left(X_{t} \in d x \mid Y_{1: t}\right)$. Now, if the system and observation models are linear Gaussian, the posteriors would also be Gaussian and can be evaluated in closed form using a Kalman filter. For nonlinear or non-Gaussian system or observation model, except in very special cases, the filter is infinite dimensional. Particle filtering is a sequential Monte Carlo technique for approximate nonlinear filtering which was first introduced in [24] as Bayesian bootstrap filtering.

Let the initial state distribution be denoted by $\pi_{0}(d x)$, the state transition kernel by $K_{t}\left(x_{t}, d x_{t+1}\right)$ and the OL given the state, by $g_{t}\left(Y_{t} \mid x_{t}\right)$. For the SSA model, the state $X_{t}=\left[v_{t}, s_{t}, \theta_{t}\right]$, the transition kernel $K_{t}$ is defined by (8) and (13) and $g_{t}$ is defined by (12). For NSSA, $X_{t}=\left[\mu_{t}, v_{t}, s_{t}, \theta_{t}\right]$ and $K_{t}$ is given by (14) and (13). The PF [24] is a recursive algorithm which produces at each time $t$, a cloud of $N$ particles $\left\{x_{t}^{(i)}\right\}_{i=1}^{N}$, whose empirical measure closely "follows" $\pi_{t}\left(d x_{t}\right)$. It also produces an approximation of the prediction distribution, $\pi_{t \mid t-1}(d x)=\operatorname{Pr}\left(X_{t} \in d x \mid Y_{1: t-1}\right)$

It starts with sampling $N$ times from the initial state distribution $\pi_{0}(d x)$ to approximate it by $\pi_{0}^{N}(d x)=$ $(1 / N) \sum_{i=1}^{N} \delta_{x_{0}^{(i)}}(d x)$ and then implements the Bayes' recursion at each time step. Now, given that the distribution of $X_{t-1}$ given observations upto time $t-1$ has been approximated as $\pi_{t-1}^{N}(d x)=(1 / N) \sum_{i=1}^{N} \delta_{x_{t-1}^{(i)}}(d x)$, the prediction step samples the new state $\bar{x}_{t}^{(i)}$ from the distribution $K_{t-1}\left(x_{t-1}^{(i)},.\right)$. The empirical distribution of this new cloud of particles $\pi_{t \mid t-1}^{N}(d x)=(1 / N) \sum_{i=1}^{N} \delta_{\bar{x}_{t}^{(i)}}(d x)$ is an approximation to the conditional probability distribution of $X_{t}$ given observations upto time $t-1$. For each particle, its weight is proportional to the likelihood of the observation given that particle, i.e., $w_{t}^{(i)}=N g_{t}\left(Y_{t} \mid \bar{x}_{t}^{(i)}\right) / \sum_{i=1}^{N} g_{t}\left(Y_{t} \mid \bar{x}_{t}^{(i)}\right)$. $\bar{\pi}_{t}^{N}(d x)=(1 / N) \sum_{i=1}^{N} w_{t}^{(i)} \delta_{\bar{x}_{t}^{(i)}}(d x)$ is then an estimate of the probability distribution of the state at time $t$ given observations uptil time $t$. We sample $N$ times with replacement from $\bar{\pi}_{t}^{N}(d x)$ to obtain the empirical estimate

\footnotetext{
${ }^{6}$ Note, the last equation $c_{t}=U_{t} * z_{t}$ holds because

$$
\begin{aligned}
c_{t} & =U_{t}{ }^{*} v_{t}=U_{t}^{*}\left[I-z_{t-1} z_{t-1}^{*}\right] z_{t} \\
& =U_{t}{ }^{*}\left[I-z_{t-1} z_{t-1}^{*}\right] C z_{t}=U_{t}{ }^{*} U_{t} U_{t}{ }^{*} z_{t}=U_{t}{ }^{*} z_{t} .
\end{aligned}
$$
}


$\pi_{t}^{N}(d x)=(1 / N) \sum_{i=1}^{N} \delta_{x_{t}^{(i)}}(d x)$. Note that both $\bar{\pi}_{t}^{N}$ and $\pi_{t}^{N}$ approximate $\pi_{t}$, but the last step is used because it increases the sampling efficiency by eliminating samples with very low weights.

\section{E. Particle Filtering Versus Extended Kalman Filtering}

We discuss here the need for a PF and why it is better than an extended Kalman filter (EKF). An EKF [35] linearizes the nonlinear system at each time instant using Taylor series and runs a Kalman filter for the linearized system. For the Taylor series approximation, to be accurate, one requires the initial guess (point about which you linearize) to be close to the actual value at every time instant. Typically, linearization is done about the predicted state. This means that one poorly estimated state will cause more error in the linearization matrices for the next prediction and this error will propagate (causing the EKF to lose track). A poorly estimated state can occur due to an outlier observation, modeling error, large system noise, or large linearization error. A PF, on the other hand, is stable under mild assumptions [36], [37], and, hence, error due to one poorly estimated state does not increase over time (and, hence, does not lead to complete loss of track).

Also, an EKF is unable to track non-Gaussian systems, in particular, systems with multimodal priors or posteriors, while a PF can. Multimodal system models are required to model a sequence of activities or multiple simultaneous activities. In particle filtering, the number of particles $N$, required to achieve a certain performance guarantee on estimation error, does not increase much with increasing dimension of the state space [25]; it depends only on the total randomness in the system. For a system which is more random (larger system noise or observation noise), the PF performance can be improved by increasing $N$.

\section{ABNORMAL ACTIVITY DETECTION}

An abnormal activity (suspicious behavior in our case) is defined as a change in the system model, which could be slow or drastic, and whose parameters are unknown. Given a test sequence of observations and a "shape activity" model, we use the change-detection strategy discussed in [30] and [6] to detect a change (observations stop following the given shape activity model). The cases of negligible observation noise (fully observed) and nonnegligible observation noise (partially observed) are discussed separately. We consider only stationary shape activities in this paper.

\section{A. Fully Observed Case}

The system is said to be fully observed when the function $h($.$) is invertible and the observation noise is zero (negligible$ compared to the system noise $n_{t}$ ). For such a test sequence, the shape dynamics of Section III-A fully defines the "shape activity model." We can evaluate the tangent coordinates of shape $\left(v_{t}\right)$ directly from the observations using (7). We use log-likelihood to test for abnormality. A given test sequence is said to be generated by a normal activity iff the probability of occurrence of its tangent coordinates using the pdf defined by (10) is large (greater than a certain threshold). Thus, the distance to activity statistic for an $L+1$ length observation sequence ending at time $t, d_{L+1}(t)$ is the negative log likelihood of the sequence of tangent coordinates of the shape of the observations (first used by us in [38]). We can test for abnormality at any time $t$ by evaluating $d_{L+1}(t)$ for the past $L+1$ frames. $d_{L+1}(t)$ is defined as follows:

$$
\begin{aligned}
d_{L+1}(t)= & -2 \log p\left(v_{t-L}, v_{t-L+1}, \ldots, v_{t}\right) \\
= & K+v_{t-L}^{T} \Sigma_{v}^{-1} v_{t-L} \\
& +\sum_{\tau=t-L+1}^{t}\left(v_{\tau}-A v_{\tau-1}\right)^{T} \Sigma_{n}^{-1}\left(v_{\tau}-A v_{\tau-1}\right)
\end{aligned}
$$

Note, here, that $\Sigma_{v}$ is always rank deficient since $\left\{v_{t}\right\}$ lie in a $(2 k-4)$-dimensional hyperplane of $\Re^{2 k}$, and, hence, the inverse defined above actually represents the pseudoinverse.

\section{B. Partially Observed Case}

In a partially observed system, the observation noise in the configuration landmarks' measurements is nonnegligible and it is defined by the observation model discussed in Section III-B. The $\mathrm{PF}$ is used to estimate the posterior distribution of shape at time $t$ given observations upto $t-1$ (prediction) and upto $t$ (filtering). We use the change-detection strategy described in [30], [6].

1) If the abnormality is a drastic one it will cause the PF, with $N$ large enough to accurately track only normal activities, to lose track. This is because under the normal activity model [(8) and (13)], the abnormal activity observations (which do not follow this model) would appear to have a very large observation noise. Thus, the tracking error (TE) will increase for an abnormal activity (very quickly for a drastic one) and this can be used to detect it. The TE or prediction error is the distance between the current observation and its prediction based on past observations, i.e.,

$$
\begin{aligned}
\mathrm{TE} \triangleq\left\|Y_{t}-\hat{Y}_{t}\right\|^{2} & =\left\|Y_{t}-E\left[Y_{t} \mid Y_{0: t-1}\right]\right\|^{2} \\
& =\left\|Y_{t}-E_{\pi_{t \mid t-1}}\left[h\left(X_{t}\right)\right]\right\|^{2} .
\end{aligned}
$$

Also, instead of TE, observation likelihood (OL) can also be used and as discussed in [6, Ch. 2], OL $\approx T E$ for white Gaussian noise.

2) For the case when the abnormality is a slow change (say a person walking away slowly in a wrong direction), the $\mathrm{PF}$ does not lose track very quickly (the TE increases slowly), or if it is a short duration change, it may not lose track at all. The TE will, thus, take longer to detect the change or it may not detect it at all. For such a case, we use the expected (negative) log likelihood (ELL) [31], [30], ELL $=E_{\pi_{t}}\left[-\log p\left(v_{t}\right)\right]$. Note that the ELL is a posterior expectation of the right-hand side of (18) with $L=0$. In general, one could use a sequence of past shapes $(L>0)$ in this case as well. The expression for $E L L$ is approximated by $\mathrm{ELL}^{N}$ as follows:

$$
\begin{aligned}
& \mathrm{ELL}^{N} \triangleq E_{\pi_{t}^{N}}\left[-\log p\left(v_{t}\right)\right]=\frac{1}{N} \sum_{i=1}^{N} v_{t}^{(i)^{T}} \Sigma_{v}^{-1} v_{t}^{(i)}+K \\
& \text { where } \quad K \triangleq-\log \sqrt{(2 \pi)^{2 k-4}\left|\Sigma_{v}\right|} .
\end{aligned}
$$




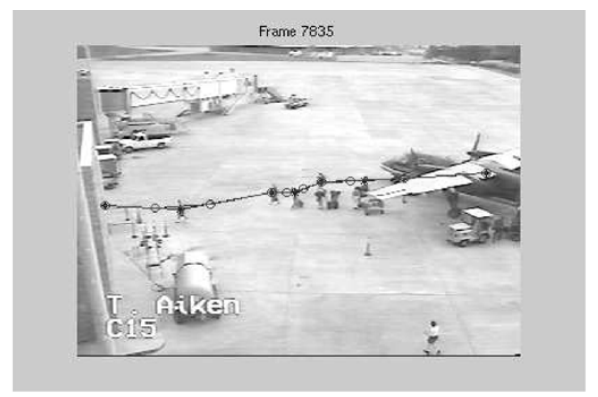

(a) A 'normal activity' frame with 4 people

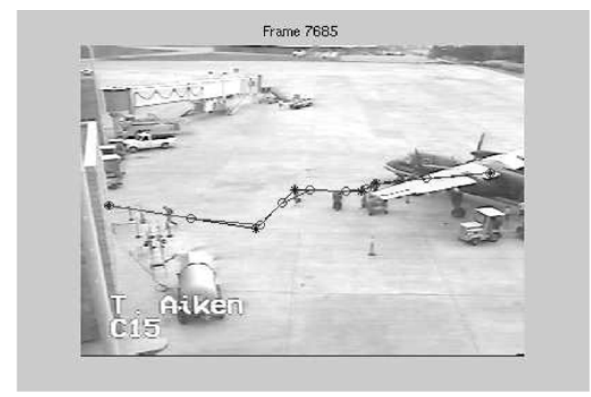

(b)Abnormality introduced by making one person walk-away in an abnormal direction

Fig. 2. Airport example: Passengers deplaning.

As explained in [6] and [30], ELL uses the tracked part of the change to detect it and hence is able to detect slow changes when they become "detectable" [30].

Thus, to detect any kind of abnormality (slow or drastic) without knowing its rate of change, we use a combination of ELL and $T E$. We declare a sequence of observations to be abnormal when either ELL or TE exceeds its corresponding threshold.

\section{TIME-VARYING NUMBER OF LANDMARKS}

All the analysis until now assumes that a configuration of points is represented as an element of $\Re^{2 k}$ where $k$ is a fixed number of landmarks. Now, we consider what happens when the number of landmarks (here the point-objects) is time varying, even though the curve formed by joining their locations remains similar. For example, a group of people (or also a group of vehicles) moving on a certain path with fixed initial and final points but number of people on the path decreases by one when a person leaves and increases by one when someone enters. In such a case, we linearly interpolate the curve by joining the landmark points in a predefined order and then resample the interpolated curve to get a fixed number of landmarks. The interpolation depends on the parametrization of the curve, which is an ill-posed problem when the data is inherently discrete. We have attempted to use two different schemes which exist in the literature: "arc-length resampling" (also known as "equidistant sampling") and "uniform resampling" which use two different parameterizations.

In "arc-length resampling," one looks at the curve formed by joining the landmarks in a predefined order and parameterizes the $x$ and $y$ coordinates by the length $l$ of the curve, upto that landmark. Let $\left[x_{t}(l), y_{t}(l)\right]$ be one-dimensional functions of the curve length and seen this way the discrete landmarks $x_{t, j}=x_{t}\left(l_{j}\right), y_{t, j}=y_{t}\left(l_{j}\right), j=0,1, \ldots k_{t}-1$ are nonuniformly sampled points from the function $\left[x_{t}(l), y_{t}(l)\right]$ with $l_{0}=0, l_{j}^{2}=l_{j-1}^{2}+\left(x_{t, j}-x_{t, j-1}\right)^{2}+\left(y_{t, j}-y_{t, j-1}\right)^{2}$. We linearly interpolate using these discrete points to estimate the function $\left[\hat{x}_{t}(l), \hat{y}_{t}(l)\right]$ and then resample it uniformly at points $\tilde{l}_{j}=(j-1) L / k, j=0,1, \ldots k-1(L$ is the total length, $L^{2}=\sum_{j} l_{j}^{2}$ ) to get a fixed number $k$ of uniformly spaced landmarks. Thus, for every configuration of $k_{t}$ landmarks, we get a new configuration of uniformly sampled (and, hence, uniformly spaced) $k$ landmarks. The linear interpolation and resampling stages can be approximated as a linear transformation
$B_{t}$ (a $k_{t} \times k$ matrix) applied to the original points. The covariance of observation noise in the resampled points becomes $\Sigma_{\mathrm{obs}, t}^{k}=B_{t} \Sigma_{\mathrm{obs}, t}^{k_{t}} B_{t}^{T}=B_{t} C^{k_{t}} \sum_{\mathrm{obs}, \mathrm{raw}, t}^{k_{t}} C^{k_{t}^{T}} B_{t}^{T}$.

"Uniform resampling," on the other hand, assumes that the observed points are uniformly sampled from some process $\left[x_{t}(s), y_{t}(s)\right]$, i.e., it assumes that the observed points are parameterized as $x_{t, j}=x_{t}\left(s_{j}\right), y_{t, j}=y_{t}\left(s_{j}\right)$ with $s_{j}=(j-1) / k_{t}$. We linearly interpolate to estimate $\left[\hat{x}_{t}(s), \hat{y}_{t}(s)\right]$ and resample it uniformly at points $\tilde{s}_{j}=(j-1) / k$ to get a fixed number of landmarks, $k$. Assuming the observed points to be uniformly sampled makes this scheme very sensitive to the changing number of landmarks. Whenever the number of landmarks changes, there is a large change in the resampled points' configuration. This leads to more false alarms while performing abnormal activity detection, but, unlike "arc-length resampling," this scheme gives equal importance to all observed points irrespective of the distance between consecutive points and so is more quick to detect abnormalities in shape caused even by two closely spaced points. We discuss an example in Section VI-D.

\section{EXPERIMENTAL AND SIMULATION RESUlTS}

\section{A. Dataset and Experiments}

We have used a video sequence of passengers deplaning and walking toward the airport terminal as an example of a "stationary shape activity." The number of people in the scene varies with time. We have resampled the curve formed by joining their locations using "arc-length resampling" (described in Section V) in all experiments except the temporal abnormality [3] detection, where we use "uniform resampling." As we needed observation noise-free data to learn the system model, we used hand-marked passenger locations for training. The mean shape $\mu$ and the tangent space Gauss-Markov model parameters, $A, \Sigma_{v}$, and $\Sigma_{n}$, were learned using this data (as discussed in Section III-A). Also, the motion model parameters (which in this case model random motion of the camera) were estimated with this data. Simulated test sequences were produced by adding observation noise to the hand-marked data. We did this to study robustness of the method to increasing observation noise. We also tested with real observations obtained using a motion-detection algorithm [34]. Both real and simulated observation sequences were tracked using the PF described in Section III-D with the number of particles $N=1000$. 


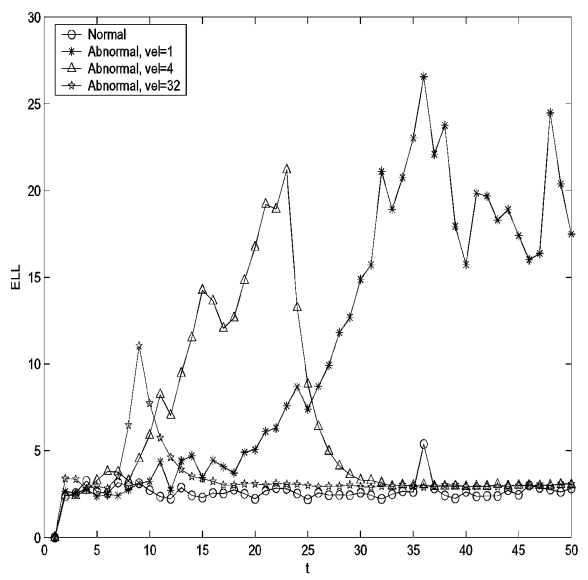

(a) ELL

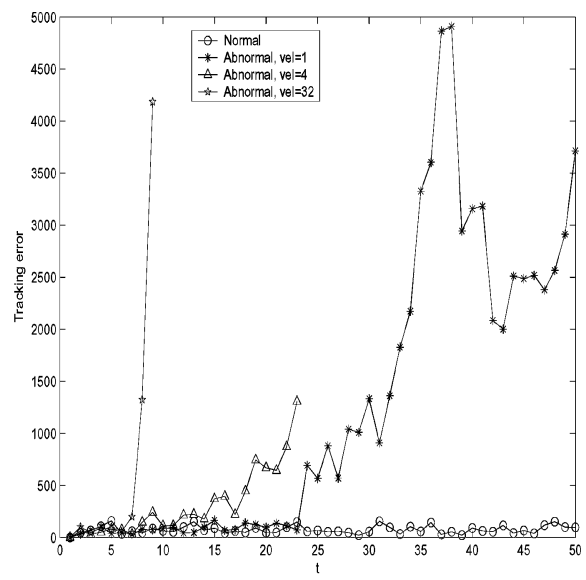

(b) Tracking error

Fig. 3. ELL and TE plots: simulated observation noise, $\sigma_{\text {obs }}^{2}=9$ (three-pixel noise).

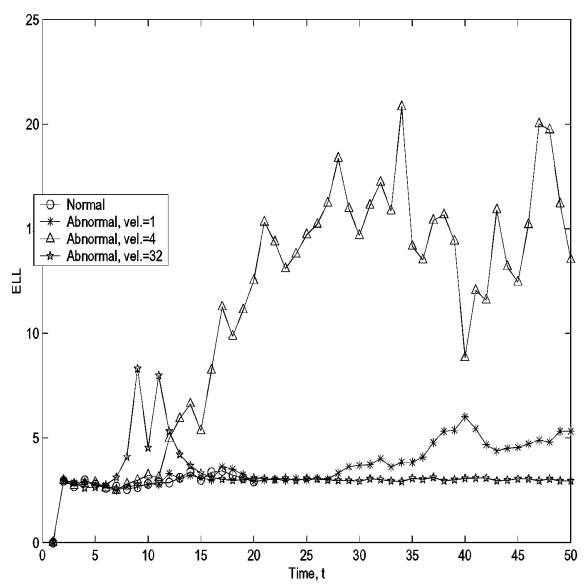

(a) ELL

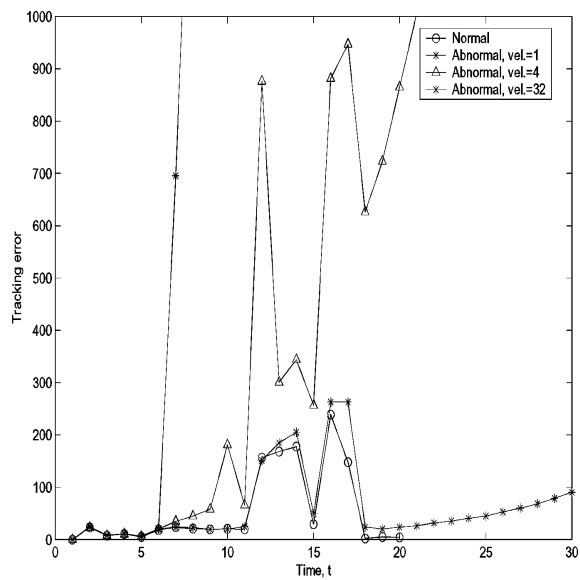

(b) Tracking error

Fig. 4. ELL and TE plots: real observations. Abnormality was introduced at $t=5$. The ELL is able to detect slow changes better while the TE works better for drastic changes. The plots are discussed in Section VI-B.

This video was provided to us by the transport security administration (TSA) and did not have any instances of abnormal behavior. Abnormal behavior was simulated in software by making one of the persons walk away in an abnormal direction (in the results shown one person was made to walk away at an angle of $45^{\circ}$ to the $X$ axis, see Fig. 2(b); Fig. 2(a) shows a normal activity frame). Now, the person could be moving away at any speed which will make the abnormality a slow or a drastic change. We have simulated this by testing for walk away speeds of $1,2,4,16$, and 32 pixels/time step in both $x$ and $y$ directions. The average speed of any person in the normal sequence is about 1 pixel/time step. Thus, walk-away velocity of $1 \mathrm{pixel} /$ time step, denoted as vel. $=1$, corresponds to a slow change, which does not go out of track for a long time while vel. $=32$ is a drastic change that causes the PF to lose track immediately.

We show change-detection results and tracks using real observations of the passengers' locations in each frame obtained using a motion-detection algorithm described in [34]. The ability of our algorithm to deal with temporal abnormalities [3] is demonstrated as well. We also plot the receiver operating characteristic (ROC) curves for change detection using the ELL, the TE and a combination of both.

\section{B. ELL Versus TE: Slow and Drastic Changes}

Fig. 3 shows ELL and TE plots for simulated observation noise and Fig. 4 shows the plots for real observations. Real observations are obtained using a motion detector [34]. Observation noise is because of the sensor noise and motion-detection error. Now, Fig. 9(b) shows a slow abnormality (vel. = 1) introduced at $t=5$ which is tracked correctly for a long time [TE plot is shown in Fig. 4(b)] and hence we need to use ELL to detect it [ELL plot is shown in Fig. 4(a)]. Fig. 9(c) shows a drastic abnormality (vel. $=32$ ] which was also introduced at $t=5$ but loses track immediately. In this case, the abnormal observations are ignored and the PF continues to follow the system model. As a result, the ELL [plot shown in Fig. 4(a)] confuses it for a normal sequence and fails completely, while TE [plot shown in Fig. 4(b)] detects it immediately. In Fig. 4(a), we show the ELL plot for increasing rates of change. With vel. $=1$, the abnormality (introduced at $t=5$ ) gets detected at $t=27$, and with vel. $=4$, it gets detected at $t=12$. For vel. $=32$, the ELL is unable to detect the abnormality. The TE [Fig. 4(b)] detects this abnormality immediately (at $t=6$ ) while it misses detecting the slow abnormality (vel. $=1$ ).

This demonstrates the need to use a combination of ELL and TE to detect both slow and drastic changes (since the aim is to 


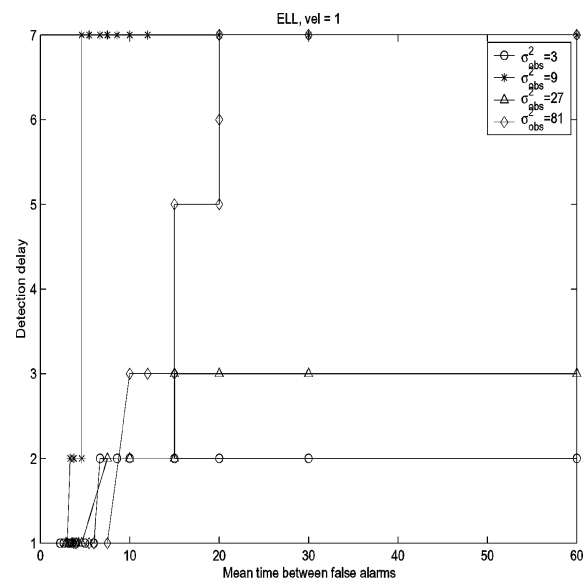

(a) Slow change (vel.=1): WORKS

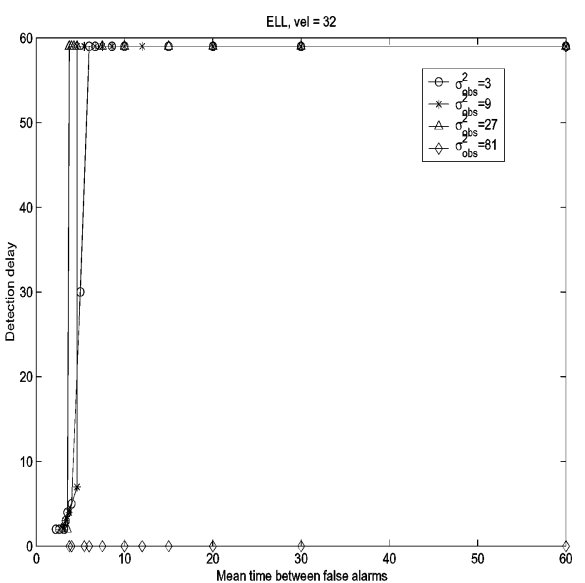

(b) Drastic change (vel.=32): FAILS

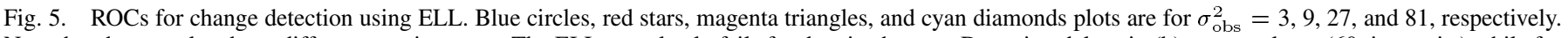

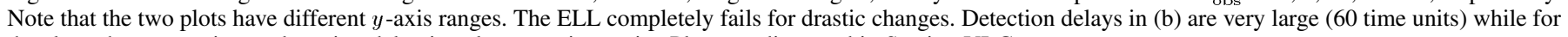
the slow-change maximum detection delay is only seven time units. Plots are discussed in Section VI-C.

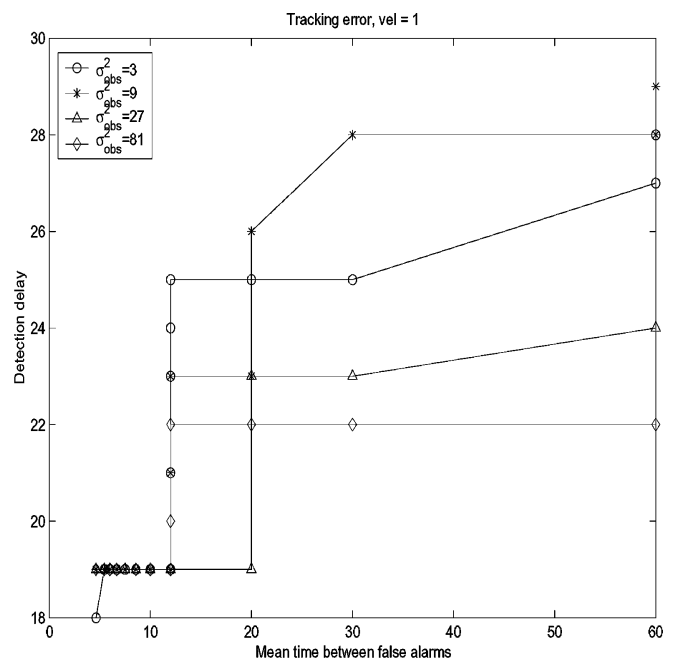

(a) Slow change (vel.=1): DOES NOT WORK

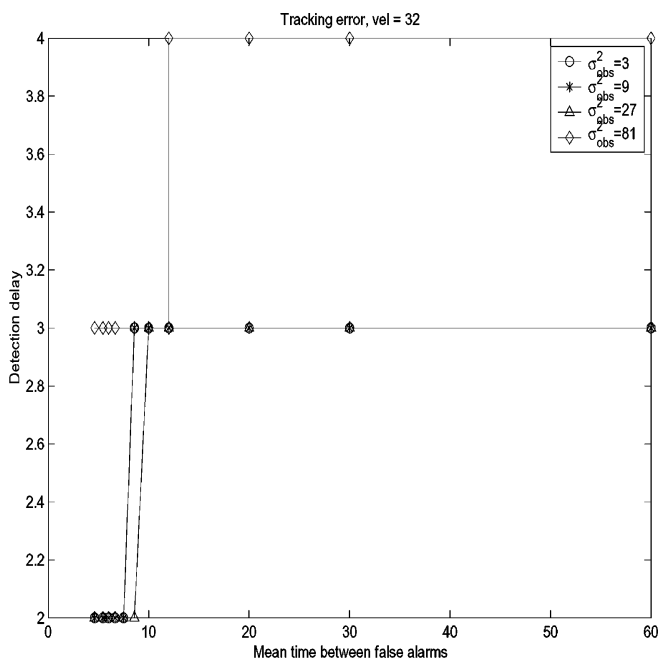

(b) Drastic change (vel.=32): WORKS

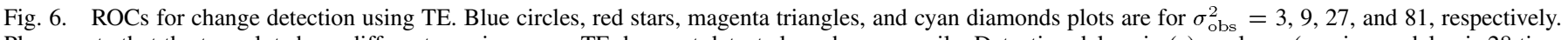

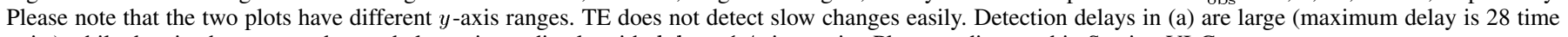
units) while drastic changes are detected almost immediately with delay $\leq 4$ time units. Plots are discussed in Section VI-C.

be able to detect any kind of abnormality with rate of change not known). As explained earlier, we declare an abnormality if either the ELL or the TE exceeds its corresponding thresholds. The ROC curves for this combined ELL/TE strategy are shown in Fig. 7. As is discussed below, by combining ELL and TE, we are able to detect all slow and drastic changes with detection delay less than seven time units.

\section{ROC Curves and Performance Degradation With Increasing Observation Noise}

The intuition discussed above is captured numerically in the ROC curves [33], [40] for change detection using ELL [Fig. 5(a) and (b) for slow and drastic changes, respectively], using TE [Fig. 6(a) and (b)], and using a combination of both [Fig. 7(a)-(d)]. Please note that every figure in the ROC plot has a different $y$-axis range. The blue circles, red stars, magenta triangles, and cyan diamonds are the ROC plots for simulated observation noise with increasing variances of $3,9,27$, and
81 square pixels. The ROC for a change-detection problem [40] plots the average-detection delay against the mean time between false alarms by varying the detection threshold. The aim of an ROC plot is to choose an operating point threshold which minimizes detection delay for a given value of mean time between false alarms.

For the slow change (vel. $=1$ ), the detection delay is much lesser using ELL than using the TE while the opposite is true for the drastic change (vel. $=32$ ). The detection performance degradation of ELL for slow change and of TE for drastic change with increasing observation noise is slow. In Fig. 5(a) (ELL for slow change), detection delay is less than or equal to two time units for $\sigma_{\text {obs }}^{2}=3$ and seven time units for $\sigma_{\text {obs }}^{2}=81$. In Fig. 6(b) (TE for drastic change), the detection delay is less than or equal to three time units for $\sigma_{\text {obs }}^{2}=3$ and four time units for $\sigma_{\text {obs }}^{2}=81$. Since the aim is to be able to detect all kinds of abnormalities (abnormality parameters are assumed not known), we propose to use a combination of the ELL and 


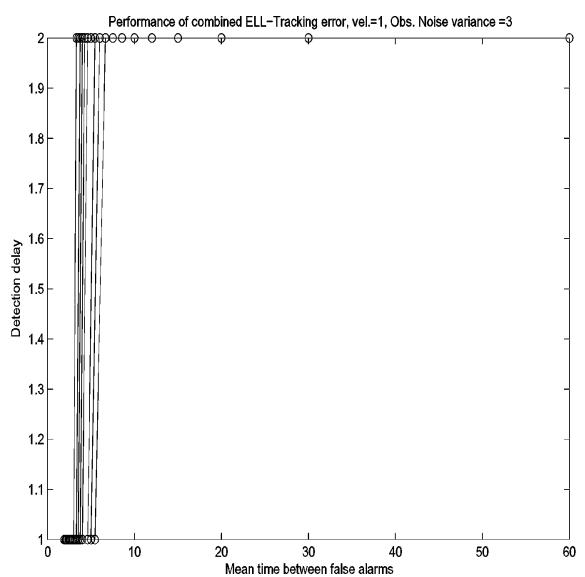

(a) $\sigma_{o b s}^{2}=3$, Slow change (vel.=1): WORKS

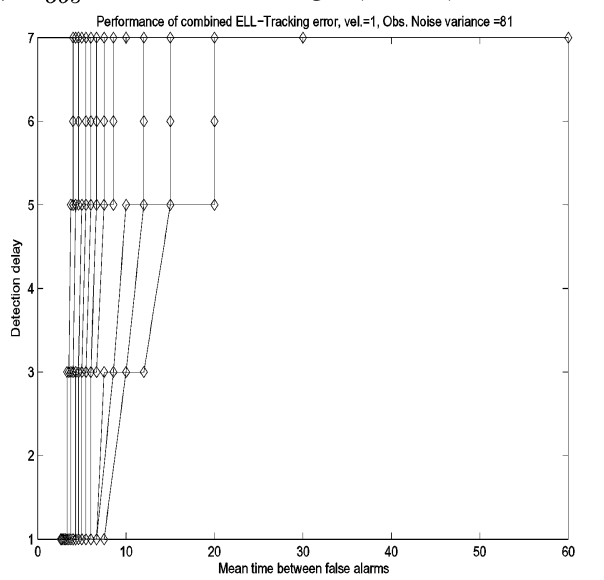

(c) $\sigma_{o b s}^{2}=81$, Slow change (vel.=1): WORKS

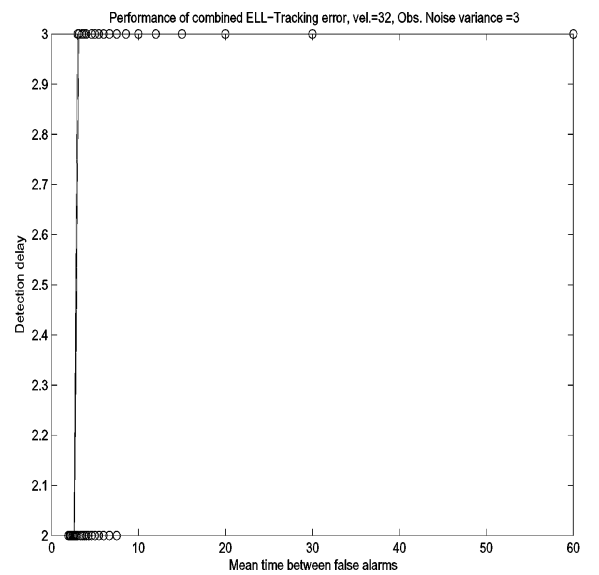

(b) $\sigma_{o b s}^{2}=3$, Drastic change (vel.=32): WORKS

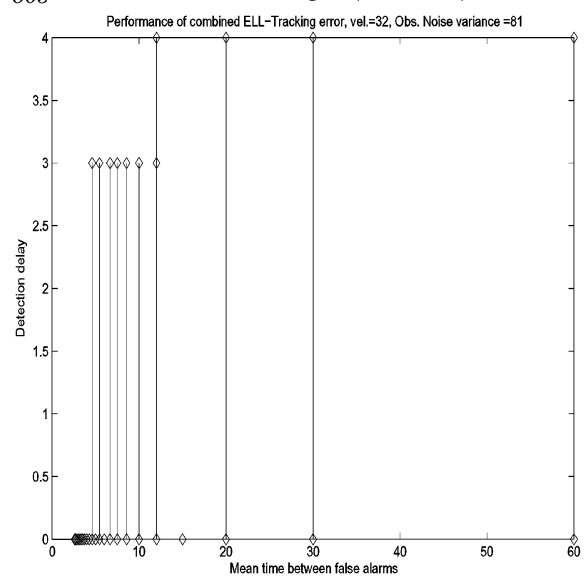

(d) $\sigma_{o b s}^{2}=81$, Drastic change (vel.=32): WORKS

Fig. 7. ROCs for change detection using the combined ELL tracking error. In this case, for each observation noise variance (in each subfigure), there are multiple curves, since one needs to vary thresholds for both ELL and TE to get the ROC. A single curve is for the ELL threshold fixed and TE threshold varying. We have a set of curves for varying ELL thresholds. The maximum-detection delay is two and three time units for $\sigma_{\text {obs }}^{2}=3$ [(a) and (b)], 7 , and 4 time units for $\sigma_{\text {obs }}^{2}=81$ $[(\mathrm{c})$ and (d)]. Plots are discussed in Section VI-C.

the TE and declare a change when either exceeds its threshold. In Fig. 7, we plot the ROC curves for slow and drastic change detection using a combination of ELL and TE. In this case, for each observation noise variance, there are multiple curves, since one needs to vary thresholds for both the ELL and the TE to get the ROC. A single curve is for the ELL threshold fixed and TE threshold varying. We have a set of curves for varying ELL thresholds. We plot the low and high observation noise cases in two separate plots. As can be seen, the combined strategy has better performance than either ELL or TE for all rates of change and for all observation noises (detection delay less than seven time units in all cases).

\section{Temporal Abnormality [3] Detection}

We also tested our method for detecting what is referred to in [3] as a temporal abnormality (one person stopped in his or her normal path). It gets detected in this framework because there is a change in shape when the person behind the stopped person goes ahead of him (curve becomes concave). We used "uniform resampling" (discussed in Section V) which detected temporal abnormality easily using ELL (Fig. 8). "Arc-length resampling" does not work too well in this case. This is because it tends to average out the locations of two closely spaced points,

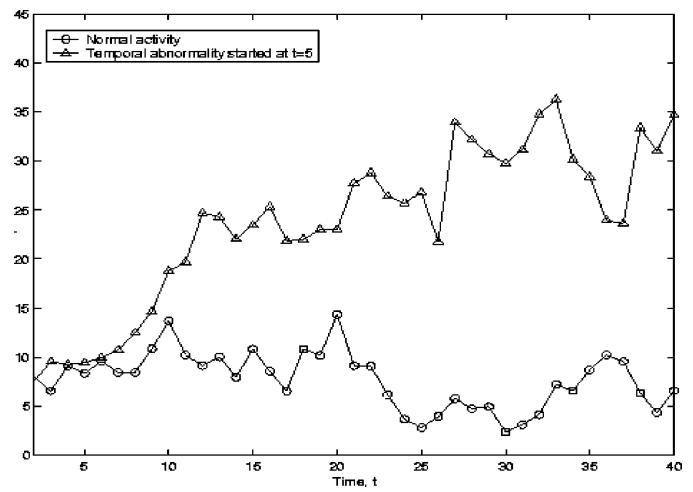

Fig. 8. ELL plot for temporal abnormality detection. Abnormality was introduced at $t=5$. The plot is discussed in Section VI-D.

thus smoothing out the concavity which needs to be detected. "Uniform resampling," on the other hand, assumes the observed points are uniformly sampled and, hence, gives equal weight to all the observed points irrespective of the distances between them. Thus, it is able to detect concavity caused even by two closely spaced points. Another way to detect temporal abnormality would be to use a NSSA model and look at deviations from the expected value of shape velocity. 


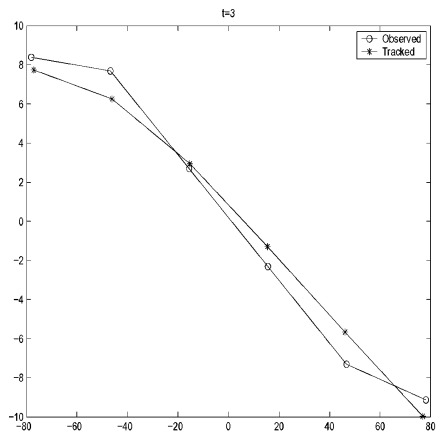

(a) Normal frame: In track

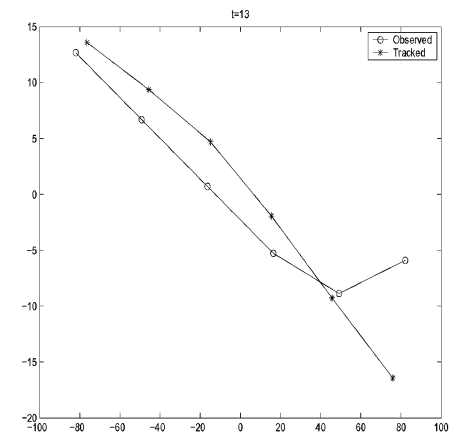

(b)Slow abnormality (vel $=1)$ : Still in track

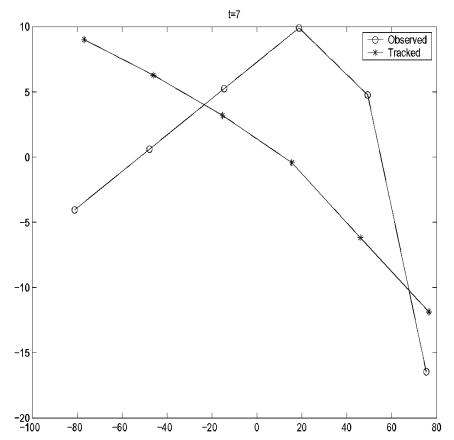

(c)Drastic abnormality (vel $=32$ ):

Loses track

Fig. 9. Tracks: real observations. Plotting the observed and tracked positions of the landmarks (passengers) on the $x-y$ plane. The plots are discussed in Section VI-E.

\section{E. Tracks}

Fig. 9(a) shows a normal observation frame (circles) and the corresponding tracked configuration (stars) for real observations obtained using a motion detector [34] on the image sequences. The observation noise was modeled to be Gaussian (although the PF can filter non-Gaussian noise, as well) and its covariance was learned from a training sequence of observations obtained using the motion detector. This shows the ability of our model to potentially be used for "tracking to obtain observations." Fig. 9(b) and (c) shows tracking of a slow (vel =1) and drastic ( vel $=32$ ) abnormality both introduced at $t=5$. As can be seen, the drastic abnormality has lost track at $t=7$ while the slow one is not totally out of track even at $t=13$. The NSSA model tracks abnormality better [6]. Note that, since we use only a point-object abstraction for moving objects (here persons), we show observed and tracked point-object locations only without showing the actual images.

\section{EXTENSIONS}

We discuss here two extensions of our work; see [6] and [39] for more details.

\section{A. Tracking to Obtain Observations [39]}

In the entire discussion until now, we used a PF in the filtering mode to estimate the probability distribution of shape from noisy observations and used this distribution for abnormality detection, but the PF also provides at each time instant the prediction distribution $\pi_{t}\left(X_{t} \mid Y_{1: t-1}\right)$, which can be used to predict the expected configuration at the next time instant using past observations, i.e., $\hat{Y}_{t} \triangleq E\left[Y_{t} \mid Y_{0: t-1}\right]=E_{\pi_{t \mid t-1}}\left[h\left(X_{t}\right)\right]$. We can use this information to improve the measurement algorithm used for obtaining the observations (a motion detector [34] in our case). Its computational complexity can be reduced and its ability to ignore outliers can be improved by using the predicted configuration and searching only locally around it for the current observation. ${ }^{7}$ As we show in Section VI-E, the observed

\footnotetext{
${ }^{7}$ One thing to note here is that, in certain cases (for example, if the posterior of any state variable is multimodal), evaluating the posterior expectation as a prediction of the current observation is not the correct thing to do. In such a case, one can track the observations using the CONDENSATION algorithm [26], which searches for the current observation around each of the possible $h\left(\bar{x}_{t}^{i}\right), i=1,2, \ldots, N$.
}

configuration is close to its prediction when there is no abnormality or change, and, hence, the prediction can be used to obtain the observation. An SSA model can track a normal activity while the NSSA is able to track abnormality as well (shown in [6]).

If used in this "tracking observations and filtering" framework, a lot of drastic abnormalities can be detected at the measurement stage itself because no observations will be found in the "vicinity" (region of search defined using observation noise variance) of the predicted position, but an outlier might get confused with a drastic abnormality since even for an outlier we will not find any observation in the "vicinity." The difference is that outliers would be temporary (one or two time instants and then the PF comes back in track), while a drastic abnormality will appear to be an outlier for a sequence of frames. Thus, by averaging the number of detects over a sequence of past time instants, we can separate outliers from real abnormalities.

Also, if the configuration is a moving one, then the predicted motion information can be used to translate, zoom, or rotate the camera (or any other sensor) to better capture the scene but in this case, one would have to alter the motion model to include a control input.

\section{B. Activity Sequence Identification and Tracking [39]}

Consider two possible situations for tracking a sequence of activities. Assume that each activity is represented by an SSA model so that the sequence of activities is characterized by a piecewise stationary shape activity model (discussed in [6]). The mean shape of each SSA component is known but the transition times are assumed unknown.

1) First, consider the simple case when there are just two possible activities and their order of occurrence is known, only the change time is unknown. In this case, one can detect the change using ELL (before the PF loses track) and then start tracking it with the second activity's transition model.

2) Now, consider the general case when a sequence of activities occur, and we do not know the order in which they occur. In this case, we can use a discrete mode variable as part of the state vector to denote each activity type. 
We make the state transition model a mixture distribution and keep the mode variable as a state. Whenever a change occurs, it takes the mode variable a few time instants to stabilize to the correct mode. One could replace the multimodal dynamics with that of the detected mode once the mode variable has stabilized. Also, in this case, we can declare an activity to be abnormal (i.e., neither of the known activity types) if the ELL w.r.t all known models exceeds a threshold.

\section{CONCLUSION AND FUTURE DIRECTIONS}

In this paper, we have presented a "shape activity model," which is a continuous-state HMM for the changing configuration of a set of moving landmarks. The shape and global motion parameters constitute the hidden-state vector and the observed landmark locations form the observation vector. The state dynamics and the mapping between the state and the observation is nonlinear, and, hence, the shape is estimated from the noisy observations using a PF. Abnormal activity detection is formulated as a change-detection problem with change parameters being unknown and change being slow or drastic. We have used a change-detection strategy using PFs which has been proposed and analyzed by us in past work [30], [31], [41]. Experimental results have been shown for abnormal activity detection in an airport scenario.

As part of future work, we hope to implement joint tracking and abnormality detection and tracking a sequence of activities (discussed in Section VII). Also, in this work, we have experimented only with stationary shape activities. We are currently studying the nonstationary case (discussed in Section III-C) in more detail. We hope to characterize (define a pdf for) specific instances of a normal activity in the nonstationary case and to define the abnormality-detection problem. The nonstationary shape activity model provides the flexibility to model and track a much larger class of group activities. We are also experimenting with a piecewise stationary shape activity model which can be used along with ELL for activity sequence segmentation and tracking.

The issue of a time-varying number of landmarks needs to be studied more rigorously by first defining the optimality criterion to make the interpolation problem well posed and then deciding the best strategy. Also, the current shape space $\left(\Re^{k m}\right.$ modulo Euclidean similarity transformations) can be replaced by general shape spaces, for example, the affine shape space ([2, Ch. 12]) would be useful to make the activity invariant to an affine camera's motion. Finally, we plan to apply our framework to many other applications (discussed in Section I).

\section{ACKNOWLEDGMENT}

The authors would like to thank F. Zhang and Prof. A. Tits of the Electrical and Computer Engineering Department and D. Jacobs of the Computer Science Department, University of Maryland, College Park, for interesting discussions and comments on the work.

\section{REFERENCES}

[1] D. Kendall, D. Barden, T. Carne, and H. Le, Shape and Shape Theory. New York: Wiley, 1999.

[2] I. Dryden and K. Mardia, Statistical Shape Analysis. New York: Wiley, 1998.

[3] W. Grimson, L. Lee, R. Romano, and C. Stauffer, "Using adaptive tracking to classify and monitor activities in a site," in Proc. IEEE Conf. Computer Vision and Pattern Recognition, Santa Barabara, CA, 1998, pp. 22-31.

[4] T. Huang, D. Koller, J. Malik, G. Ogasawara, B. Rao, S. Russell, and J. Weber, "Automatic symbolic traffic scene analysis using belief networks," in Proc. American Association for Artificial Intelligence Conf., 1994, pp. 966-972.

[5] J. Spletzer, A. Das, R. Fierro, C. Taylor, V. Humar, and J. Ostrowski, "Cooperative localization and control for multi-robot manipulation," presented at the Conf. Intelligent Robots and Systems, 2001.

[6] N. Vaswani, "Change detection in stochastic shape dynamical models with applications in activity modeling and abnormality detection," $\mathrm{Ph} . \mathrm{D}$ dissertation, Elect. Comput. Eng. Dept., Univ. Maryland, College Park, 2004.

[7] C. Zahn and R. Roskies, "Fourier descriptors for plane closed curves," IEEE Trans. Comput., vol. C-21, no. 3, pp. 269-281, Mar. 1972.

[8] D. F. Rogers and J. A. Adams, Mathematical Elements for Computer Graphics. New York: WCB/McGraw-Hill, 1990.

[9] T. Cootes, C. Taylor, D. Cooper, and J. Graham, "Active shape models: Their training and application," Comput. Vis. Image Understand., vol. 61, no. 1, pp. 38-59, Jan. 1995.

[10] - "Training models of shape from sets of examples," in Proc. Brit. Machine Vision Conf., 1992, pp. 9-18.

[11] J. Kent, "The complex bingham distribution and shape analysis," J. Roy. Stat. Soc., Ser. B, pp. 56:285-299, 1994.

[12] Y. Zhou, L. Gu, and H. Zhang, "Bayesian tangent space model: Estimating shape and pose parameters via Bayesian inference," presented at the IEEE Conf. Computer Vision and Pattern Recognition, Madison, WI, Jun. 2003.

[13] C. Small, The Statistical Theory of Shape. New York: Springer, 1996.

[14] A. Srivastava and E. Klassen, "Geometric filtering for subspace tracking," Adv. Appl. Probl., vol. 36, no. 1, Mar. 2004.

[15] A. Chiuso and S. Soatto, "Monte-Carlo filtering on Lie groups," presented at the IEEE Conf. Decision and Control, Sydney, Australia, Dec. 2000.

[16] S. Kurakake and R. Nevatia, "Description and tracking of moving articulated objects," in Proc. Int. Conf. Pattern Recognition, The Hague, Netherlands, Aug. 1992, pp. I:491-495.

[17] T. Starner and A. Pentland, "Visual recognition of American sign language using hidden Markov models," presented at the Int. Workshop on Face and Gesture Recognition, 1995.

[18] C. Bregler, "Learning and recognizing human dynamics in video sequences," in Proc. IEEE Conf. Computer Vision and Pattern Recognition, 1997, pp. 568-574.

[19] A. Bobick and Y. Ivanov, "Action recognition using probabilistic parsing," in Proc. IEEE Conf. Computer Vision and Pattern Recognition, Santa Barbara, CA, 1998, pp. 196-202.

[20] A. Roy Chowdhury and R. Chellappa, "A factorization approach for event recognition," presented at the CVPR Event Mining Workshop, Madison, WI, Jun. 2003.

[21] L. Torresani and C. Bregler, "Space-time tracking," presented at the Eur. Conf. Computer Vision, Copenhagen, Denmark, May 2002.

[22] L. Zelnik-Manor and M. Irani, "Event based analysis of video," presented at the IEEE Int. Conf. Computer Vision, Vancouver, BC, Canada, 2001.

[23] D. P. T. Syeda-Mahmood, "Recognizing action events from multiple viewpoints," presented at the IEEE Workshop on Detection and Recognition of Events in Video, Vancouver, BC, Canada, Jul. 2001

[24] N. Gordon, D. Salmond, and A. Smith, "Novel approach to nonlinear/nonGaussian Bayesian state estimation," Proc. Inst. Elect. Eng., pp. 140(2):107-113, 1993

[25] A. Doucet, N. deFreitas, and N. Gordon, Sequential Monte Carlo Methods in Practice. New York: Springer, 2001.

[26] J. MacCormick and A. Blake, "A probabilistic contour discriminant for object localization," presented at the IEEE Int. Conf. Computer Vision (ICCV), Mumbai, India, Jan. 1998.

[27] S. Zhou and R. Chellappa, "Probabilistic human recognition from video," in Proc. Eur. Conf. Computer Vision, Copenhagen, Denmark, May 2002, pp. 681-697. 
[28] D. Schulz, W. Burgard, D. Fox, and A. Cremers, "Tracking multiple moving targets with a mobile robot using particle filters and statistical data association," presented at the IEEE Int. Conf. Robotics and Automation (ICRA), Seoul, Korea, May 2001.

[29] B. Azimi-Sadjadi and P. Krishnaprasad, "Change detection for nonlinear systems: A particle filtering approach," presented at the Amer. Control Conf., Anchorage, AK, May 2002.

[30] N. Vaswani, "Change detection in partially observed nonlinear dynamic systems with unknown change parameters," presented at the Amer. Control Conf., Boston, MA, Jun. 2004.

[31] N. Vaswani, A. Roy Chowdhury, and R. Chellappa, "Activity recognition using the dynamics of the configuration of interacting objects," presented at the IEEE Conf. Computer Vision and Pattern Recognition, Madison, WI, Jun. 2003.

[32] A. J. Yezzi and S. Soatto, "Deformotion: Deforming motion, shape average and the joint registration and approximation of structures in images," Int. J. Comput. Vis., vol. 53, no. 2, pp. 153-167, 2003.

[33] A. Papoulis, Probability, Random Variables and Stochastic Processes. New York: McGraw-Hill, 1991.

[34] Q. Zheng and S. Der, "Moving target indication in LRAS3 sequences," presented at the 5th Annu. Fedlab Symp., College Park, MD, 2001.

[35] T. Kailath, A. Sayed, and B. Hassibi, Linear Estimation. Upper Saddle River, NJ: Prentice-Hall, 2000.

[36] D. Crisan and A. Doucet, "A survey of convergence results on particle filtering for practitioners," IEEE Trans. Signal Process., vol. 50, no. 3, pp. 736-746, May 2002.

[37] F. LeGland and N. Oudjane, "Stability and uniform approximation of nonlinear filters using the Hilbert metric, and application to particle filters," INRIA, Tech. Rep., RR-4215, 2002.

[38] N. Vaswani, A. Roy Chowdhury, and R. Chellappa, "Statistical shape theory for activity modeling," presented at the IEEE Int. Conf. Acoustics, Speech, and Signal Processing, Apr. 2003.

[39] N. Vaswani and R. Chellappa, "Nonstationary shape activities," presented at the IEEE Conf. Decision and Control, Seville, Spain, Dec. 2005.

[40] M. Basseville and I. Nikiforov, IEEE Conference on Decision and Control. Upper Saddle River, NJ: Prentice-Hall, 1993.

[41] N. Vaswani, "Bound on errors in particle filtering with incorrect model assumptions and its implication for change detection," presented at the IEEE Int. Conf. Acoustics, Speech, and Signal Processing, Montreal, QC, Canada, May 2004.

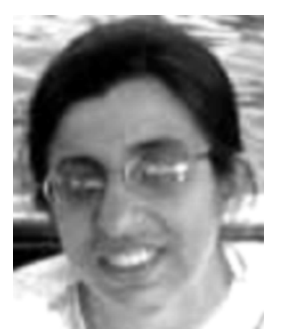

Namrata Vaswani (M'99) received the B.Tech. degree in electrical engineering from the Indian Institute of Technology (I.I.T.), Delhi, in 1999, and the $\mathrm{Ph} . \mathrm{D}$. degree in electrical and computer engineering from the University of Maryland, College Park, in August 2004. Her Ph.D. thesis was on change detection in stochastic shape dynamical models and applications to activity modeling and abnormal activity detection.

She was a Postdoctoral Fellow with the School of Electrical and Computer Engineering, Georgia Institute of Technology, Atlanta, from 2004 to 2005, where she worked on particle filtering algorithms for level-set representations of continuous curves and their applications to tracking deformable objects. She is currently an Assistant Professor with the Department of Electrical and Computer Engineering, Iowa State University, Ames. Her research interests are in detection and estimation problems in signal and video processing, computer vision, and in biomedical image processing. In particular, she is interested in particle filtering theory and applications in tracking and change detection and in shape analysis and filtering. In the past, she has also worked on subspace methods for image classification.

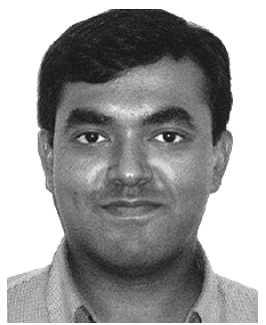

Amit K. Roy-Chowdhury received the B.S. degree in electrical Engineering from Jadavpur University, Calcutta, India, in 1985, the M.S. degree in engineering in systems science and automation from the Indian Institute of Science, Bangalore, in 1997, and the Ph.D. degree from the Department of Electrical and Computer Engineering, University of Maryland, College Park, in 2002, where he worked on statistical error characterization of 3-D modeling from monocular video sequences.

$\mathrm{He}$ is an Assistant Professor in the Electrical Engineering Department, University of California, Riverside. He was previously with the Center for Automation Research, University of Maryland, as a Research Associate. He was involved in projects related to face, gait, and activity modeling and recognition. His research interests are in signal, image and video processing, computer vision, and pattern recognition.

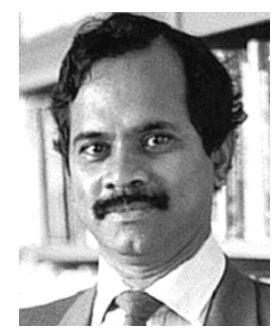

Rama Chellappa (S'78-M'79-SM'83-F'92) received the B.E. (Hons.) degree from the University of Madras, Madras, India, in 1975 and the M.E. (Distinction) degree from the Indian Institute of Science, Bangalore, in 1977. He received the M.S.E.E. and $\mathrm{Ph} . \mathrm{D}$. degrees in electrical engineering from Purdue University, West Lafayette, IN, in 1978 and 1981, respectively.

Since 1991, he has been a Professor of electrical engineering and an affiliate Professor of Computer Science at the University of Maryland, College Park. Recently, he was named the Minta Martin Professor of Engineering. He is also affiliated with the Center for Automation Research (Director) and the Institute for Advanced Computer Studies (permanent member). Prior to joining the University of Maryland, he was an Assistant Professor (1981 to 1986) and an Associate Professor (1986 to 1991) and Director of the Signal and Image Processing Institute (1988 to 1990) with the University of Southern California (USC), Los Angeles. Over the last 24 years, he has published numerous book chapters and peer-reviewed journal and conference papers. He has edited a collection of Papers on Digital Image Processing (Los Alamitos, CA: IEEE Computer Society Press, 1992), coauthored a research monograph on Artificial Neural Networks for Computer Vision (with Y. T. Zhou) (New York: Springer-Verlag, 1990), and co-edited a book on Markov Random Fields: Theory and Applications (with A. K. Jain) (New York: Academic, 1993). His current research interests are face and gait analysis, 3-D modeling from video, automatic target recognition from stationary and moving platforms, surveillance and monitoring, hyperspectral processing, image understanding, and commercial applications of image processing and understanding.

Dr. Chellappa has served as an Associate Editor of the IEEE TRANSACTIONS on Signal Processing, IEEE TRansactions on Pattern ANALYsis and Machine InTELligence, IEEE TRANSACTIONS ON IMAGe PROCESSING, and IEEE Transactions on Neural Networks. He was Co-Editor-in-Chief of Graphical models and Image Processing. He is now serving as the Editor-in-Chief of the IEEE TRANSACTIONS ON PATTERN ANALYSIS AND MACHINE INTELLIGENCE. He served as a member of the IEEE Signal Processing Society Board of Governors from 1996 to 1999. Currently, he is serving as the Vice President of Awards and Membership for the IEEE Signal Processing Society. He has served as a General the Technical Program Chair for several IEEE international and national conferences and workshops. He received several awards, including the National Science Foundation (NSF) Presidential Young Investigator Award, an IBM Faculty Development Award, the 1990 Excellence in Teaching Award from School of Engineering at USC, the 1992 Best Industry Related Paper Award from the International Association of Pattern Recognition (with Q. Zheng), and the 2000 Technical Achievement Award from the IEEE Signal Processing Society. He was elected as a Distinguished Faculty Research Fellow (1996 to 1998) at the University of Maryland, he is a Fellow of the International Association for Pattern Recognition, and he received a Distinguished Scholar-Teacher award from the University of Maryland in 2003. 